FN. Low BMD was significantly correlated with increased age $(p=0.01)$ and disease duration $(p=0.05)$, post-menopausal status $(p=0.04)$, higher BMI $(p=0.004)$, musculoskeletal involvement $(p=0.01)$, association to other rheumatic diseases $(p=0.01)$, higher disease activity by SLEDAI score $(p=0.05)$, higher Erythrocyte sedimentation Rate $(p=0.01)$ and C-Reactive Protein $(p=0.007)$, low serum complement C3 $(p=0.009)$ and C4 $(p=0.04)$ and cumulative doses of GCs $(p=0.01)$. We found also that BMD at LS was mostly affected by GCs intake, BMI and CRP while BMD at FN was mostly affected by SLEDAI score, C3 and C4 ( $<<0.001$ in all cases). Gender, history of smoking and cardiovascular comorbidities had no significant impact on BMD.

Conclusion: OP is a common but unrecognised complication of SLE with increased frequency of both peripheral and vertebral FFx. Our study suggests a high risk profile for OP and FFx in SLE which seems to be associated with age, disease duration, post-menopausal status, BMI and GCs.

REFERENCES:

[1] Dey, M., \& Bukhari, M. (2018). Predictors of fracture risk in patients with systemic lupus erythematosus. Lupus, 27(9), 1547-1551.

Disclosure of Interests: None declared

DOI: 10.1136/annrheumdis-2021-eular.3717

\section{AB0340 PATIENTS WITH LABORATORY CRITERIA OF ANTIPHOSPHOLIPID SYNDROME AND "NON- CRITERIA" MANIFESTATIONS: A MULTICENTER STUDY}

G. Pires Da Rosa ${ }^{1,2}$, E. Ferreira ${ }^{2,3}$, B. Sousa-Pinto ${ }^{4,5}$, P. Bettencourt ${ }^{2,6}$, R. Cervera', G. Espinosa' ${ }^{1}{ }^{1}$ Hospital Clínic, Institut d'Investigacions Biomèdiques August Pi I Sunyer (IDIBAPS), University of Barcelona, Department of Autoimmune Diseases, Barcelona, Catalonia, Spain; ${ }^{2}$ University of Porto, Faculty of Medicine, Porto, Portugal; ${ }^{3}$ Centro Hospitalar Universitário de São João, Department of Internal Medicine, Porto, Portugal; ${ }^{4}$ CINTESIS Center for Health Technology and Services Research, Porto, Portugal; ${ }^{5}$ Faculty of Medicine, University of Porto, MEDCIDS - Department of Community Medicine, Information and Health Decision Sciences, Porto, Portugal; ${ }^{6}$ Hospital CUF, Department of Internal Medicine, Porto, Portugal

Background: Patients with laboratory criteria for antiphospholipid syndrome (APS) and presenting only "non-criteria" clinical manifestations are scarcely described in the literature [1-3].

Objectives: To describe a cohort of these patients, portraying potential associations between "non-criteria" manifestations, antiphospholipid antibodies (aPL) and treatments.

Methods: Retrospective analysis of patients with aPL positivity fulfilling APS criteria assessed in two tertiary European hospitals between 2005 and 2020. Those without criteria clinical manifestations but with the presence of "non-criteria" manifestations were included.

Results: A total of 75 patients were analyzed. The predominant non-obstetric involvements were hematologic (thrombocytopenia, hemolytic anemia) and "mild" neurological (white matter lesions, migraine), with an absence or near absence of cutaneous, cardiac, pulmonary and more severe neurologic manifestations (Table 1). In obstetric morbidity, two spontaneous abortions before 10 weeks and infertility were the more frequent manifestations. LA positivity was positively associated with non-obstetric manifestations (OR=4.2, 95\% Cl 1.5-11.9; $\mathrm{p}=0.007$ ) while an aCL profile was associated with obstetric manifestations (OR 7.0, 95\% $\mathrm{Cl}$ 2.3-21.3; $\mathrm{p}=0.001$ ). A suspicion of progression towards SLE was considered plausible in $13(19 \%)$ of non-SLE individuals. Twelve $(40.0 \%)$ of patients with thrombocytopenia as their only hematological involvement required corticosteroids. History of infertility (OR $6.1,95 \% \mathrm{Cl} 1.3-28 ; \mathrm{p}=0.02$ ) and IVF recurrent failure (OR $6.5,95 \% \mathrm{Cl} 1.2-36.3 ; \mathrm{p}=0.032$ ) were associated with pregnancy treatment with LMWH/LDA combination. Among patients under anticoagulation, clinical manifestations included brain white matter lesions, APS nephropathy, superficial vein thrombosis, amaurosis fugax, and livedoid vasculopathy. A higher value of GAPSS was observed in patients with non-obstetric (median 9, IQR 5-13) in comparison to those with obstetric manifestations (median 5, IQR 4-5.5). Conclusion: The present article stresses the clinical relevance of these patients, with an impression that at least some manifestations will contribute in the future to the classification of individuals as definite APS.

REFERENCES:

[1] J. Alijotas-Reig et al., Comparative study of obstetric antiphospholipid syndrome (OAPS) and non-criteria obstetric APS (NC-OAPS): report of 1640 cases from the EUROAPS registry. Rheumatology (Oxford), (2019).

[2] M. Fredi et al., Risk Factors for Adverse Maternal and Fetal Outcomes in Women With Confirmed aPL Positivity: Results From a Multicenter Study of 283 Pregnancies. Front Immunol 9, 864 (2018).

[3] E. Sevim et al., Characteristics of Antiphospholipid Antibody Positive Patients in AntiPhospholipid Syndrome Alliance for Clinical Trials and InternatiOnal Networking. Arthritis Care \& Research n/a.
Table 1. "Non-criteria" clinical manifestations present in the analyzed population.

\begin{tabular}{lc}
\hline Clinical manifestation & $\mathbf{n}(\%)$ \\
\hline Non-obstetric & $\mathbf{n}=\mathbf{4 9}$ \\
\hline Thrombocytopenia & $34(69.4)$ \\
Coombs' positivity & $11(22.4)$ \\
Migraine & $11(22.4)$ \\
Hemolytic anemia & $5(10.2)$ \\
Brain MRI white matter lesions & $5(10.4)$ \\
Raynaud's phenomenon & $5(10.2)$ \\
Superficial vein thrombosis & $4(8.1)$ \\
Evans syndrome & $4(8.1)$ \\
Amaurosis fugax & $3(6.1)$ \\
APS nephropathy & $2(4.1)$ \\
Livedo reticularis & $1(2.0)$ \\
Livedoid vasculopathy & $1(2.0)$ \\
Memory lapses & $1(2.0)$ \\
Pseudo-multiple sclerosis & $1(2.0)$ \\
Sensorineural hearing loss & $1(2.0)$ \\
Splinter hemorrhages & $1(2.0)$ \\
Obstetric & $\mathbf{n}=38$ \\
Two spontaneous abortions <10 weeks & $16(42.1)$ \\
Infertility & $14(36.8)$ \\
$\geq 2$ or more IVF failures & $11(28.9)$ \\
Late IUGR (>34 weeks) & $7(18.4)$ \\
Premature birth between 34 and 37 weeks & $7(18.4)$ \\
Placental ischemia & $5(13.1)$ \\
Late preeclampsia (>34 weeks) & $3(8.0)$ \\
Placental abruption & $2(5.3)$ \\
Placental hematoma & $2(5.3)$ \\
\hline Abbreviations -aCL: a & \\
\hline
\end{tabular}

Abbreviations -aCL: anticardiolipin antibodies; Al: Autoimmune; AID: Autoimmune disease; aPL: Antiphospholipid antibodies; IUGR: Intrauterine growth restriction; IVF: in vitro fertilization; LA: Lupus anticoagulant.

Disclosure of Interests: None declared

DOI: 10.1136/annrheumdis-2021-eular.3757

\section{AB0341 SLE PREGNANCIES AT HIGH RISK FOR PRE- ECLAMPSIA BENEFIT MOST FROM COMBINATION OF LOW DOSE ASPIRIN AND HYDROXYCHLOROQUINE}

$\underline{\text { I. Haase }}^{1}$, G. Chehab ${ }^{1}$, O. Sander ${ }^{1}$, M. Schneider ${ }^{1}$, R. Fischer-Betz ${ }^{1} .{ }^{1}$ HeinrichHeine-University Duesseldorf, Policlinic for Rheumatology \& Hiller Research Unit for Rheumatology, Duesseldorf, Germany

Background: Women with SLE face a high risk of preeclampsia (PE). Low dose Aspirin (LDA) is known to protect against $P E$ in non-autoimmune patients and is recommended for SLE patients ${ }^{1}$. Besides, a beneficial effect of hydroxychloroquine $(\mathrm{HCQ})$ on the occurrence of $P E$ has recently been discussed ${ }^{2}$.

Objectives: To investigate the influence of LDA and HCQ on the occurrence of PE in SLE patients.

Methods: Pregnancies of women with SLE from an outpatient pregnancy clinic were prospectively evaluated. Clinical characteristics, medication use and preg nancy outcomes were analysed. Association of LDA use (latest from gestationa week 16 on) and $\mathrm{HCQ}$ use (from $1^{\text {st }}$ trimester on) with PE were analysed using a multiple logistic regression model (adjustment for age, BMI, hypertension, disease activity in $1^{\text {st }}$ trimester, lupus nephritis, nulliparity, history of PE and highrisk aPL profile ${ }^{3}$ ).

Results: We enrolled 190 lupus pregnancies (148 women, 1995-2019). Additional risk factors for PE were present in $83.7 \%: 55.8 \%$ showed a high-risk profile (HRP) for PE according to $\mathrm{ACOG}^{4}$ (history of $\mathrm{PE}$, hypertension, lupus nephritis or $\mathrm{aPL}$ ), another $27.9 \%$ had at least one moderate risk factor (nulliparous, BMI>30 or age $>35$ ) (see table 1).

Each $20.5 \%$ of pregnancies received $\mathrm{HCQ}$ only or LDA only, while $22.6 \%$ were prescribed both drugs. 36.3\% took neither HCQ nor LDA. Women with HRP were more likely to take LDA alone or in combination with $\mathrm{HCQ}(28.3 \%$ and $35.8 \%$, respectively).

PE occurred in $13.2 \%$ of pregnancies $(7.7 \%$ in HCQ only, $15.4 \%$ in LDA only, $14.0 \%$ in $\mathrm{HCQ}$ and LDA, $14.5 \%$ in no $\mathrm{HCQ}$ or LDA). Most pregnancies affected by PE carried a HRP (88.0\%).

Use of LDA was significantly associated with a lower risk for PE [aOR 0.21 (95\%$\mathrm{Cl}$ 0.05-0.99), $\mathrm{p}<0.05]$. $\mathrm{HCQ}$ use also had a moderating effect on the incidence of $\mathrm{PE}$, but this effect did not reach significance [aOR 0.47 (95\%-Cl 0.15-1.52) $\mathrm{p}=0.21$ ]. If only pregnancies with HRP were considered, the effect size increased for HCQ (LDA [aOR 0.18 (95\%-Cl 0.04-0.96), p<0.05], HCQ [aOR 0.28 (95\%-Cl 0.07-1.14), $p=0.075]$ ).

Conclusion: In this prospective real-life cohort, timely LDA medication was associated with a lower risk of PE in SLE pregnancies. In a multiple regression model taking LDA and HCQ into account, the favourable effect on the occurrence of $\mathrm{PE}$ was partially explained by $\mathrm{HCQ}$. In particular, SLE patients at high risk 
for PE seem to benefit from HCQ during pregnancy. Future research may reveal mechanisms by which $\mathrm{HCQ}$ might lower the incidence of PE.

REFERENCES:

[1] doi: 10.1136/annrheumdis-2016-209770

[2] doi: $10.1111 / \mathrm{bcp} .14131$

[3] doi: 10.1136/annrheumdis-2019-215213

[4] doi: 10.1097/AOG.0000000000002708

Table 1. Patient characteristics and risk profile

\begin{tabular}{|c|c|c|c|c|c|}
\hline & & $\begin{array}{l}\text { HCQ only } \\
\quad(n=39)\end{array}$ & $\begin{array}{l}\text { LDA only } \\
\qquad(n=39)\end{array}$ & $\begin{array}{c}\mathrm{HCQ}+\mathrm{LDA} \\
(\mathrm{n}=43)\end{array}$ & $\begin{array}{c}\text { no HCQ } \\
\text { or LDA } \\
(n=69)\end{array}$ \\
\hline \multirow[t]{4}{*}{$\begin{array}{l}\text { Patient } \\
\text { characteritics }\end{array}$} & $\begin{array}{l}\text { Age (years), median } \\
\text { (IQR) }\end{array}$ & $29(25-33)$ & $32(30-33)$ & $30(28.5-33.5)$ & $31(28-35)$ \\
\hline & BMI, median (IQR) & $23.5(22-25)$ & $23(22-24)$ & $22(22-24)$ & $23(22-24)$ \\
\hline & $\begin{array}{l}\text { Chronic Hypertension, } \\
\text { n (\%) }\end{array}$ & 7 (17.9\%) & $9(23.1 \%)$ & $6(14.0 \%)$ & $9(13.0 \%)$ \\
\hline & $\begin{array}{c}\text { Preconception counsel- } \\
\text { ling, } \mathrm{n}(\%)\end{array}$ & $23(59.0 \%)$ & $28(71.8 \%)$ & $35(81.4 \%)$ & $41(59.4 \%)$ \\
\hline \multirow{3}{*}{$\begin{array}{l}\text { Obstetrical } \\
\text { history }\end{array}$} & Nulliparous, n (\%) & $20(51.3 \%)$ & $26(66.7 \%)$ & $33(76.7 \%)$ & $36(52.2 \%)$ \\
\hline & $\begin{array}{c}\text { Previous fetal loss, } \\
\mathrm{n}(\%)\end{array}$ & $1(2.6 \%)$ & $10(25.6 \%)$ & $14(32.6 \%)$ & $10(14.5 \%)$ \\
\hline & Previous PE, n (\%) & $2(5.1 \%)$ & $4(10.3 \%)$ & $4(9.3 \%)$ & $5(7.2 \%)$ \\
\hline \multirow[t]{6}{*}{$\begin{array}{l}\text { SLE } \\
\text { characteristics } \\
\text { and therapy }\end{array}$} & $\begin{array}{c}\text { Disease duration } \\
\text { (years), median } \\
\text { (IQR) }\end{array}$ & $7.0(2.0-11.0)$ & $7.5(3.0-11.5)$ & $6.7(3.4-10.0)$ & $6.0(3.0-9.2)$ \\
\hline & SLEDAI, median (IQR) & $2.0(1.0-4.3)$ & $2.0(0-2.5)$ & $4.0(2.0-4.0)$ & $2.0(0-4.0)$ \\
\hline & Lupus nephritis, n (\%) & $9(23.1 \%)$ & $13(33.3 \%)$ & $18(41.9 \%)$ & $14(20.3 \%)$ \\
\hline & $\begin{array}{c}\text { Anti-dsDNA antibodies, } \\
\mathrm{n}(\%)\end{array}$ & $22(56.4 \%)$ & $15(38.5 \%)$ & $36(83.7 \%)$ & $33(47.8 \%)$ \\
\hline & $\begin{array}{l}\text { Prednisolone therapy, } \\
\qquad \mathrm{n}(\%)\end{array}$ & $26(66.7 \%)$ & $15(38.5 \%)$ & $25(58.1 \%)$ & $29(42.0 \%)$ \\
\hline & $\begin{array}{c}\text { Prednisolone }(\mathrm{mg} / \mathrm{d}) \text {, } \\
\text { median }(\mathrm{IQR})\end{array}$ & $5.0(5.0-8.0)$ & $5.0(5.0-7.5)$ & $5.0(4.0-5.0)$ & $5.0(5.0-8.0)$ \\
\hline \multirow[t]{5}{*}{ aPL status } & APS, n (\%) & - & $12(30.8 \%)$ & $14(32.6 \%)$ & $2(2.9 \%)$ \\
\hline & Any positive aPL, n (\%) & - & $20(51.3 \%)$ & 19 (44.2\%) & $5(7.2 \%)$ \\
\hline & LAC, $n(\%)$ & - & 15 (38.5\%) & $13(30.2 \%)$ & $3(4.3 \%)$ \\
\hline & $\mathrm{ACL}, \mathrm{n}(\%)$ & - & $14(35.9 \%)$ & $13(30.2 \%)$ & $4(5.8 \%)$ \\
\hline & $\beta 2-G P 1, n(\%)$ & - & $12(30.8 \%)$ & $12(27.9 \%)$ & $2(2.9 \%)$ \\
\hline
\end{tabular}

$\mathrm{LAC}=$ Lupus anticoagulant, $\mathrm{ACL}=$ Anticardiolipin antibody, $\beta 2-\mathrm{GP} 1=\beta 2$-Glycoprotein I antibody

Disclosure of Interests: None declared

DOI: 10.1136/annrheumdis-2021-eular.3839

\section{AB0342 SYNDROME: ARE THEY DIFFERENT ENTITIES?}

R. Serrano Morales ${ }^{1}$, R. Nieto ${ }^{1}$, S. Porta ${ }^{1}$, K. Roberts ${ }^{1}$, L. Lema ${ }^{1}$,

C. Maldini ${ }^{1}$, Y. Tissera ${ }^{1}$, J. A. Albiero ${ }^{1}$, V. Savio ${ }^{1}$, C. Goizueta ${ }^{1}$, V. Lobo ${ }^{1}$,

A. Broll ${ }^{1}$, N. Cucchiaro', S. "perez ${ }^{1}$, P. Izaguirre ${ }^{1}$, A. Estevez ${ }^{1}$, A.

I. Abalo ${ }^{1}$, M. Etcheverry', V. I. Bellomio ${ }^{1}$, M. L. Leguizamón ${ }^{1}$, N. Antoniol ${ }^{1}$,

C. Perandones ${ }^{1}$, C. Pisoni ${ }^{1}$, M. L. De la Torre ${ }^{1}$, M. García ${ }^{1}$, M. S. Velloso ${ }^{1}$,

M. Sacnun ${ }^{1}$, C. Gobbi ${ }^{1}$, D. Guaglione ${ }^{1}$, F. Vivero ${ }^{1}$, V. Saurit ${ }^{1}$, N. Benzaquen ${ }^{1}$,

V. Nemman ${ }^{1}$, G. Remondino ${ }^{1}$, M. Machado Escobar ${ }^{1}$, M. Scolnik',

C. Danielsen ${ }^{1}$, G. Gomez ${ }^{1}$, A. M. Babini ${ }^{1}$, A. Brigante ${ }^{1}$, A. Luissi ${ }^{1}$, V. V. Castro

Coello $^{1}$, L. Sorrentino ${ }^{1}$, H. Berman ${ }^{1}$, P. Alba ${ }^{1}$, G. Pons-Estel ${ }^{1}$ on behalf of

Antiphospholipid Syndrome Study GroupArgentine Society of Rheumatology.

${ }^{1}$ Argentine Society of Rheumatology, APS Study Group, Buenos Aires,

Argentina

Background: Several studies showed two main clinical phenotypes of antiphospholipid syndrome (APS): thrombotic (TAPS) and obstetric APS (OAPS). Although they have the same autoantibody profile, one of them developed thrombosis and other one obstetric morbidity.

Objectives: To study clinical, demographic and antibody profile in patients with TAPS and OAPS.

Methods: we retrospectively evaluated TAPS and OAPS patients who were included in Argentine Antiphospholipid antibodies registry. We studied clinical, demographic and antibody profile in both groups.

Results: 238 patients were included in the registry. 201 (84.81\%) of them were female. $122(60.69 \%)$ of them fullfilled APS Sydney classification criteria, 47 (38.52\%) TAPS and $52(42.62 \%)$ OAPS. 23 (18.85\%) patients had both thrombotic and obstetric events so they were excluded in this analysis.

Arterial Hypertension (HBP) and Hyperlipidemia were more frequent in TAPS versus OAPS. Older age was found in TAPS as well as in association with Systemic lupus erythematosus (SLE). There was no difference in antibody profile between the 2 groups, and the Global Antiphospholipid Syndrome Score (aGAPSS) was higher in TAPS than OAPS.
$18(38.3 \%)$ of TAPS patients had at least 1 pregnancy. Mean number of pregnancies of TAPS was $2.5(1.10)$ and 3.84 (1.86) in OAPS. Thrombotic events were not found in TAPS during pregnancy and puerperium. HBP and gestational diabetes (GD) and other pregnancy related comorbidities were found in TAPS.

\begin{tabular}{|c|c|c|c|c|c|c|c|c|}
\hline & $\begin{array}{l}\text { OAPS } \\
(n=52)\end{array}$ & $\begin{array}{l}\text { TAPS } \\
(n=47)\end{array}$ & $\mathrm{P}$ & & $\begin{array}{l}\text { OAPS } \\
(n=52)\end{array}$ & & $\begin{array}{l}\text { TAPS } \\
(n=18)\end{array}$ & \\
\hline SLE, $n(\%)$ & $\begin{array}{c}11 \\
(21.2)\end{array}$ & 28 (59.6) & 0.0002 & & $\mathrm{~N}$ & $\%(D E)$ & $\mathrm{N}$ & $\%(D E)$ \\
\hline $\begin{array}{l}\text { aGAPSS, } \\
\quad \text { mean (RIQ) }\end{array}$ & $4(5)$ & $8(5)$ & $<0.0001$ & $\begin{array}{c}\text { Abortions }(<10 \\
\text { weeks) }\end{array}$ & 33 & 38.4 (36.2) & 8 & $\begin{array}{l}26.5 \\
(35.3)\end{array}$ \\
\hline $\begin{array}{l}\text { Age, mean } \\
\quad(D E)\end{array}$ & $\begin{array}{c}39.3 \\
(6.24)\end{array}$ & $\begin{array}{c}43.1 \\
(13.5)\end{array}$ & $<0.0001$ & Live Birth & 36 & $33.5(28.1)$ & 16 & $\begin{array}{l}72.2 \\
(34.7)\end{array}$ \\
\hline HBP, $n(\%)$ & $5(9.6)$ & 15 (31.9) & 0.0121 & $>37$ weeks & 21 & $16.4(2.38)$ & 16 & 61.1 \\
\hline $\begin{array}{l}\text { Hyperlipidemia, } \\
n(\%)\end{array}$ & $4(7.7)$ & $12(25.5)$ & 0.0267 & $\begin{array}{c}\text { Prematurity } \\
<37>34 \\
\text { weeks }\end{array}$ & 11 & $8.76(1.97)$ & 0 & - \\
\hline $\mathrm{GD}, n(\%)$ & $3(5.8)$ & $2(4.3)$ & 0.9999 & $\begin{array}{l}\text { Prematurity } \\
<34 \text { weeks }\end{array}$ & 9 & $6.37(1.49)$ & 1 & $\begin{array}{l}1.39 \\
(5.89)\end{array}$ \\
\hline $\begin{array}{l}\text { Obesity, } \\
n(\%)\end{array}$ & $8(15.4)$ & $4(8.5)$ & 0.2912 & $\begin{array}{c}\text { Pre eclampsia } \\
>34-<37 \\
\text { weeks }\end{array}$ & 1 & $\begin{array}{l}0.490 \\
(0.0350)\end{array}$ & 2 & $\begin{array}{l}4.63 \\
(0.138)\end{array}$ \\
\hline $\begin{array}{l}\text { Smoking, } \\
n(\%)\end{array}$ & $\begin{array}{c}11 \\
(21.1)\end{array}$ & 13 (27.6) & 0.4019 & $\begin{array}{l}\text { Placental } \\
\text { Hematoma }\end{array}$ & 3 & $1.86(8.18)$ & 1 & $2.78(11.8)$ \\
\hline $\begin{array}{l}\text { Sedentary life- } \\
\text { style, } n(\%)\end{array}$ & $\begin{array}{c}16 \\
(30.8)\end{array}$ & 17 (36.2) & 0.8486 & $\begin{array}{l}\text { Abruptio } \\
\text { Placentae }\end{array}$ & 2 & $1.96(9.80)$ & 2 & $\begin{array}{l}4.17 \\
(12.9)\end{array}$ \\
\hline Triple Positivity & 5 & $\begin{array}{c}4 \\
8.5\end{array}$ & 0.8323 & $\begin{array}{l}\text { Normal } \\
\text { delivery }\end{array}$ & 20 & $17.8(27.9)$ & 14 & $\begin{array}{l}47.7 \\
(33.9)\end{array}$ \\
\hline $\begin{array}{l}\text { Double } \\
\text { Positivity }\end{array}$ & 1 & 3 & & $\begin{array}{r}\text { Cesarean } \\
\text { section }\end{array}$ & 22 & $17.2(21.6)$ & 2 & $11.1(32.3)$ \\
\hline $\begin{array}{l}\text { Simple } \\
\text { Positivity }\end{array}$ & $\begin{array}{c}29 \\
38.5\end{array}$ & 40.4 & & $\begin{array}{l}\text { Urgent } \\
\text { Cesarean } \\
\text { section } \\
\text { GD } \\
\text { HBP }\end{array}$ & $\begin{array}{l}2 \\
6\end{array}$ & $\begin{array}{c}1.37(7.49) \\
3.46(10.3)\end{array}$ & $\begin{array}{l}1 \\
3\end{array}$ & $\begin{array}{c}12.5 \\
(24.6) \\
\\
2.78(11.8) \\
10.2 \\
(26.3)\end{array}$ \\
\hline
\end{tabular}

Conclusion: Antibody profile was similar in TAPS and OAPS. However, clinical manifestations and cardiovascular risk were different. These results should be evaluated in prospective studies.

Disclosure of Interests: None declared

DOI: 10.1136/annrheumdis-2021-eular.3842

\begin{tabular}{l|l}
\hline AB0343 & THE CHARACTERISTICS OF T CELLS IN SYSTEMIC \\
LUPUS ERYTHEMATOSUS PATIENTS WITH ANXIETY \\
BASED ON MACHINE LEARNING
\end{tabular}

X. Gu${ }^{1}$, Y. Jin ${ }^{1}$, R. Li', D. Zhang ${ }^{1}$, C. Dong ${ }^{1}$, Q. Zhang ${ }^{2}$, Z. Xue ${ }^{2}$, Z. Gu' ${ }^{1}$ Affiliated Hospital of Nantong University, Department of Rheumatology, Nantong, China; ${ }^{2}$ Affiliated Hospital of Nantong University, Research Center of Clinical Medicine, Nantong, China

Background: Systemic lupus erythematosus (SLE) is an autoimmune disease, the immune system of patients to be disordered, especially in $T$ cell subsets ${ }^{1}$. They were prone to mental diseases, anxiety particularly, which lead to suicide ${ }^{2}$. The recent study had reported that $C D 4^{+} \mathrm{T}$ cells in the peripheral blood played the key role in like anxiety behavior of mice ${ }^{3}$ Although there showed that the level of serum TNF- $\alpha$ in SLE patients with anxiety was higher than without anxiety ${ }^{4}$, finding the important special mediators especially in T cell subsets was still necessary for the prevention of anxiety in SLE patients.

Objectives: In total, 108 SLE patients, which met the diagnostic criteria of the American Society of rheumatology (v1997), were enrolled in this study from Affilliated Hospital of Nantong University, China. Exclusion criteria included other autoimmune diseases and active infection (including hepatitis B or C virus, Epstein-Barr virus, human immunodeficiency virus or Mycobacterium tuberculosis infection).

Methods: We surveyed the abundance of 74 immune cell subpopulations from 108 SLE patients using flow cytometry, and investigated their differences between patients with and without anxiety ( 24 versus 84 ). Moreover, machine learning including Lasso regression, Random forest (RF) and Sparsity partial least squares discriminant analysis (SPLS-DA) was employed to build models and futher selected important features for the classification of SLE patients with anxiety.

Results: SLE patients with anxiety showed higher body mass index (BMI) and lower quality of life. In their peripheral blood, the proportion of internal cell subsets composition of Th cell and Treg cells changed. By machine learning, we 